\title{
Testing Validity of Using Sample Mean in Studies of Behavioral Facts
}

\author{
Yongchen Zou, Runqi Hu \\ School of Finance and Statistics, East China Normal University, Shanghai, China \\ E-mail: rustyzou@gmail.com,rqhu712@gmail.com \\ Received Janarury18, 2011; revised April 15, 2011; accepted April 28, 2011
}

\begin{abstract}
In this paper we argue that a couple of taken-for-granted methods employed in studying behavioral facts of human risk preference are mistakable. We call for within-subjects experiment design and propose a simple statistical method that might be used to test the validity of using sample mean in interpreting as well as generalizing risk preferences.
\end{abstract}

Keywords: Risk Preferences, Behavioral Economics, Prospect Theory, Heuristics

\section{Introduction}

Decision making under uncertainty involves monetary decision between a certain amount of gain/loss and a gamble which gives you a certain chance to win the prize /lose the money, taking the form of "Choose between a sure gain/loss of $x$ or a $p$ chance to win/lose $y$ ", where $y$ is always of greater magnitude a number than $x$. An enormous amount of efforts have been invested into this field by a great many behavioral economists and psychologists, who produced various views and theories on the mechanics of mankind's decision making under uncertainty.

Pioneering problems of this kind were first presented by two psychologists, Daniel Kahneman and Amos Tversky [1] in their renowned work of prospect theory, revealing that people are generally loss averse and gain certainty-seeking. Challenging the Subjective Expected Utility (SEU) theory, prospect theory replaced the utility function with the S-shaped value function, probability with the weighting function. This critical step forward in economics received enormous attentions.

Although theories on decision making under uncertainty varies, they generally share methods such as through questionnaires responses provided by agents. Despite the many insightful views on how people make decisions, little attention has been placed on the validity of methods. We find that the ways in which data is analyzed in studies of this field are imperfect.

The main purpose of this paper is to aware of defects lurking in the methods used in studies of decision mak- ing under uncertainty, especially the misuse of sample means, and introduce an approach of experiment data analysis.

The remainder of this paper is organized as follows: Section 2 provides a review of decision making under uncertainty. Section 3 points out two major fallacies exist in the studies of this field. Section 4 presents our experiment on human risk preferences toward possible gains and losses and introduces a new approach in analyzing data that circumvents the fallacies. Section 5 proposes a theory of testing validity of use of sample average in deriving regression models. Section 6 is a conclusion.

\section{Decision under Risk}

Precursors in studies of human decisions under risk, Kahneman and Tversky concocted series of questions, and through responds from the agents they found an inclined pattern in which people make monetary decision under uncertainty. Typical problems presented by prospect theory are as follows:

Problem 1: Imagine you are now $\$ 1000$ richer. Choose between:

A. Sure gain of $\$ 500$; B. $50 \%$ chance to win $\$ 1000$

Problem 2: Imagine you are now $\$ 2000$ richer. Choose between:

C. Sure loss of $\$ 500$; D. $50 \%$ to lose $\$ 1000$

For the matter that the majority of respondents chose $\mathrm{A}$ and $\mathrm{D}$, and that $\mathrm{A}$ and $\mathrm{C}$ (as well as $\mathrm{B}$ and $\mathrm{D}$ ) are in fact technically identical-namely if people choose A (B), 
they should have chosen C (D) to stay consistent in their decision - a law in human decision making seemingly obvious arises that people tend to seek certainty in gains and averse to risk when it comes to losses, as is concluded by prospect theory. Also, people focus on gains and losses, which means that they take the reference point of zero, or status quo, rather than concerning about the final situation they will be in. For instance, if people choose $\mathrm{A}$ and $\mathrm{C}$, they will end up in both problem with a final situation of $\$ 1500$ richer.

Kahneman and Tversky (1979) replaced the utility function with what they called value function, as is plotted in Figure 1. Concave in the domain of gains and more steeply convex in that of losses, the function indicates that people are loss averse.

In addition, the two psychologists plotted a non-linear weighting function, advanced in their later work (Kahneman and Tversky, 1992), that indicating people's distortion in interpreting probability. See Figure 2. Particularly, Kahneman and Tversky asserted, people overestimate very low probability, which justifies people's actions

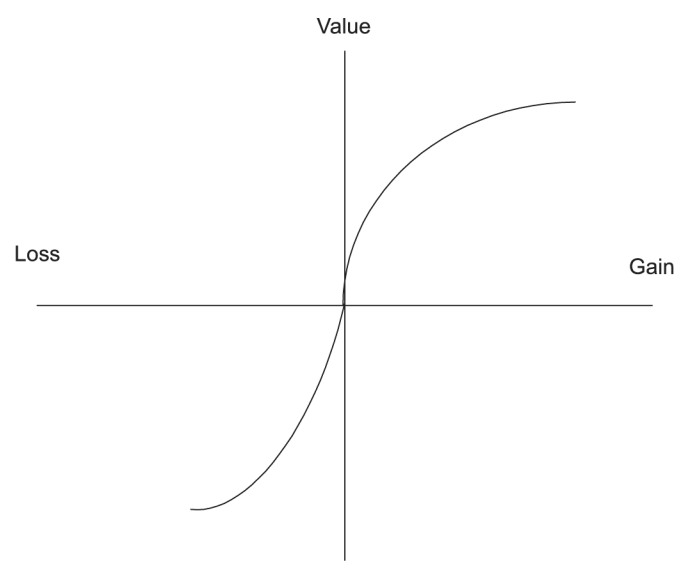

Figure 1. Prospect theory value function.

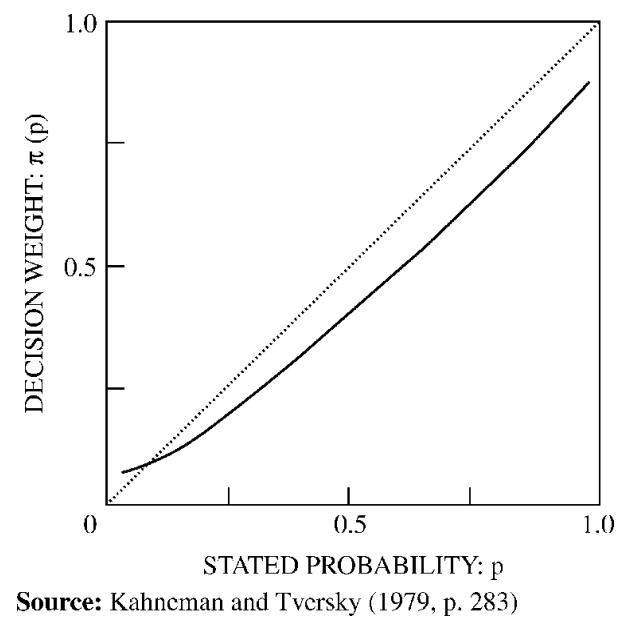

Figure 2. Prospect theory weighting function. in gambling and buying insurance. This argument is supported by results of the following problems in their 1979 paper:

Problem 3: $0.1 \%$ to win $\$ 5000$ or a certain gain of $\$ 5$ where most people choose the former, and

Problem 4: $0.1 \%$ to lose $\$ 5000$ or a sure loss of $\$ 5$ where the majority favors the latter.

Similar brief reviews of prospect theory value function could be found in Terrance Odean [2], Richard H. Thaler and Eric J. Johnson [3], Richard Thaler [4], Daniel Kahneman and Mark W. Riepe [5].

The Striking of prospect theory is a tipping point where a great amount of intellectual powers are evoked and invest their time and efforts into exploring of human decision making under risk. But as prototypes, pair choices in the original paper left much to be desired: 1) vast majority of the problems used in the paper is concerned about monetary gains and losses. And things, when associated with money, always become more complicated than they are under other situations in that special parts of people's mind will be activated by sway of human nature, such as greediness and fear of embarrassment for winning the least (losing the most) among peers; 2) all problems in that paper is hypothetical, which means that respondents won't actually win the prizes or suffer the losses, whatever choices they make. This could give rise to differences in decision making, thus leading to errors in statistics, as Steven J. Kachelmeier and Mohamed Shehata [6] proved by their experiments conducted in China, U.S., and Canada, which, however, Amos Tversky and Daniel Kahneman [7] asserted insufficient and unnecessary; 3) a potential assumption hard to be detected in the original paper is that, it presume choice makers have "reset buttons" on their body, and after every single choice is made, respondents will press the button, reset themselves to the status quo physically and psychologically, and go on with the next question. This presumption is flawed in that in a series of decision makings, risk-taking behavior is affected by the previous choices the choice maker made. Risk-taking behavior alters when previous gains or losses are incorporated into decision making. This has been discussed by Staw [8], Laughhunn and Payne [9], Tvesky and Kahneman [10], Fredrick [11].

\section{Fallacies in Methods}

Although theories of decision making vary, they generally share methods of experimental nature usually involving subjects, questionnaires, instructions, and interpretation of subjective responses. But economics, distinguished from psychology, is not experimental. The idea of generalizing what is said by myopic experiment re- 
sults into a theory of distant future has itself suffered from cognitive biases. We find that discussion of experiments results in studies of behavioral economics were long done under a couple of major assumptions, both have much to do with the use of sample mean, which require justification.

\subsection{Majority Heuristic}

Consider the process in which prospect theory value function is plotted: problem sets were concocted, choices were made by the subjects, and an S-shaped function was plotted in accordance with the majority's risk preferences. What are majority's preferences? In studies of decision making such as prospect theory, they generally refer to the options favored by most people. But we should be careful in deriving quantitative conclusion, such as the value function, because it is constituted with two parts of gain and loss domains. We argue that the intuitively natural approach of connecting two branches up needs reconsideration.

To illustrate the issue, we suppose the following two problems and the results of them, mimicking the birth of value function. Note that the case is intensely simplified.

Problem 7. Imagine you are now $\$ 2000$ richer. Choose between:

A. Sure gain of $\$ 500$; B. $50 \%$ chance to win $\$ 1000$ $[70]^{*}$

Problem 8: Imagine you are now $\$ 3000$ richer. Choose between:

C. Sure loss of $\$ 500$; D. $50 \%$ to lose $\$ 1000$

Assume that 100 subjects are involved in this study. Note that the numbers in parentheses indicate the percentage of the subjects' responses (e.g. $70 \%$ of the respondents choose A over B). Now responses of Problem 7 shows a sign of risk aversion, while those of Problem 8 indicates loss aversion. A function concave in the domain of gains and convex in the domain of losses is therefore produced, as is shown in Figure 1, representing the preference of the majority.

Here the fallacy emerges. It has long been overconfidently assumed that those who constitute the majority in Problem 7 stay the majority in Problem 8. That is, experimenters mistakingly think that most, if not all, of the 70 subjects who chose A in Problem 7 chose D in Problem 8, which is obviously erroneous for there exists intersection in choices among different options. It is reasonable to call this logic fallacy majority heuristic, and somehow evade cognitive guard.

Consider an extreme case of the responses of Problem 7 and 8: of the 70 subjects who chose A in Problem 7, only 35 D in Problem 8; and all 30 subjects who chose B in Problem 7 favored D in Problem 8. This means that the majority in Problem 8 is constituted by 35 MAJOR subjects and 30 MINOR subjects in Problem 7 whose responses were against the "trend". Similarly, of 70 subjects who chose A, 35 of them had chose C in Problem 8. We maintain that studies in this field could change for the better if results are interpreted in an innovative and more statistically reliable way such that choices made are documented, rather than marginally, in an integrate way. For instance, of a problem set constituted by Problem 7 and Problem 8, instead of merely recording the percentages of subjects who chose $\mathrm{A}, \mathrm{B}, \mathrm{C}$, and $\mathrm{D}$, more precise preferences of every individual are documented as the percentages of subjects choosing $\mathrm{AC}, \mathrm{AD}, \mathrm{BC}$ and $\mathrm{BD}$. Note that in the extreme case mentioned, the percentages were $35 \%$ for $\mathrm{AD}, 35 \%$ for $\mathrm{AC}, 30 \%$ for $\mathrm{BD}$, and $0 \%$ for BC.

To prove that the majority heuristic matters, we draw four typical problems (see Appendix) in the Kahneman and Tversky's 1979 paper and replicated the experiment in which 32 subjects are involved. Rather than merely analyzing the average preference, we recorded the result individually, and compare each of them to the average outcome.

Percentages in the parentheses shows the ratio of number of subject who made the corresponding choice to the sample size.

Note that prospect theory value function indicates two preferences of human decision making, namely risk averse in the domain of gains and risk seeking in losses. We note them R.A. in gains and R.S. in losses respectively. Now subjects are said to be R.A. in gains if they choose B in both PROB1 and PROB3, and R.S. in losses if $\mathrm{A}$ are preferred in both PROB2 and PROB4. Define a trend as existing if more than $50 \%$ of the subjects have it. A perfect subject whose preference is consistent with that is predicted by prospect theory will answer "B A B A" in the two pair of problems.

Table 1 tabulates the result of this replication experiment. We first draw an average trend of the result by counting how many people chose $\mathrm{A}$ in the four questions and then divide the numbers by the sample size. It turns out that, as can be seen in the table, in the first pair of problems, nearly $43.8 \%$ subjects chose A in PROB1, which means $55.2 \%$ chose $\mathrm{B}$, and $53 \%$ subjects chose $\mathrm{A}$ in PROB2. Thus the average trend is "B A", indicating R.A. in gains and R.S. in losses. In the second pair of problems, $81.3 \%$ answered $\mathrm{B}$ in PROB3, and $40.6 \%$ answered $\mathrm{A}$ in PROB4. This, then, represents an average trend of "B B", being R.A. in both gains and losses domains (note that this is a trend slightly disagree with prospect theory's prediction).

If we just look at the average trends, we see at least 
Table 1. Result of a replication experiment of four prospect theory problems.

\begin{tabular}{|c|c|c|c|c|}
\hline Subject & PROB1 & PROB2 & PROB3 & PROB4 \\
\hline 1 & B & A & $\mathrm{B}$ & A \\
\hline 2 & A & B & B & $\mathrm{A}$ \\
\hline 3 & B & A & B & B \\
\hline 4 & B & B & $\mathrm{B}$ & B \\
\hline 5 & B & A & $\mathrm{B}$ & B \\
\hline 6 & A & A & $\mathrm{B}$ & A \\
\hline 7 & A & A & B & A \\
\hline 8 & B & A & B & B \\
\hline 9 & A & A & B & B \\
\hline 10 & $\mathrm{~A}$ & B & $\mathrm{B}$ & B \\
\hline 11 & A & B & A & B \\
\hline 12 & A & B & A & B \\
\hline 13 & $\mathrm{~A}$ & $\mathrm{~A}$ & B & B \\
\hline 14 & B & B & A & B \\
\hline 15 & B & A & B & A \\
\hline 16 & B & A & B & A \\
\hline 17 & B & B & $\mathrm{A}$ & A \\
\hline 18 & B & B & B & A \\
\hline 19 & B & B & $\mathrm{B}$ & A \\
\hline 20 & B & A & B & A \\
\hline 21 & B & B & B & $\mathrm{B}$ \\
\hline 22 & A & A & B & B \\
\hline 23 & B & B & B & B \\
\hline 24 & A & B & $\mathrm{B}$ & B \\
\hline 25 & B & A & B & A \\
\hline 26 & A & A & B & B \\
\hline 27 & $\mathrm{~A}$ & A & A & B \\
\hline 28 & A & B & B & B \\
\hline 29 & B & B & B & B \\
\hline 30 & B & A & A & B \\
\hline 31 & A & B & B & A \\
\hline 32 & B & A & B & A \\
\hline Choose A & $14[43.75 \%]$ & 17 [53.13\%] & 6 [18.75\%] & $13[40.63 \%]$ \\
\hline Choose BA & \multicolumn{2}{|c|}{$10[31.25 \%]$} & \multicolumn{2}{|c|}{12 [37.5\%] } \\
\hline $\begin{array}{l}\text { Choose } \\
\text { BA,BA }\end{array}$ & \multicolumn{4}{|c|}{6 [18.75\%] } \\
\hline
\end{tabular}

one of them is consistent with S-shaped value function, and one might argue that if the sample size (or the number of questions) expands, prospect theory prediction could be proved right. However, if the result of this replication experiment is interpreted in a different way, the conclusion would be widely contradicting.

Instead of calculating an average answer, we now look at within-subjects, or integrate, results by checking how many subjects answered "B A" in both pair of questions, and recall that "B A" indicates a preference of R. A. in gains and R.S. in losses. As is shown in Table 1, only $31.3 \%$ and $37.5 \%$ of subjects answered "B A" in the pairs of questions respectively, a result far cry in indicating the trend of R.A. in gains and R.S. in losses. And the majority heuristic is proved existing here because although the average result shows a trend of R.A. and R.S. in the first pair of questions, it is actually supported by only $31.3 \%$ of the subjects.

Remarkably, this paradox becomes more irony when we try to find "perfect subject" who answered "B A B A": there are only six of them, namely $18.8 \%$ of the sample. If a trend is buttressed by no more than $20 \%$ of the subjects, it can never be called a trend.

We argue that the majority heuristic has taught us a paradoxical lesson that, people might be risk averse in gains; people might be risk seeking in losses; but it would be erroneous to assert that people are risk averse in gains and risk seeking in losses. As a matter of fact, we are to show in section III that even in the domains of gains and losses respectively, human preferences cannot be simply generalized as risk averse or risk seeking.

We should however point out that intersection of preferences doesn't happen all the time. Think of another two problems and a pair of possible results:

Problem 9. Choose between:

A. Sure gain of $\$ 500$; B. $50 \%$ chance to win $\$ 1000$

$$
[70]^{*}
$$

Problem 10: Choose between:

C. Sure gain of $\$ 600$; D. $50 \%$ chance to win $\$ 1,000$ $[75]^{*}$

It is reasonable in this case to predict that all those who chose $\mathrm{A}$ in the former problem favored $\mathrm{C}$ in the latter - no intersection happens. We speculate two pairs of principles in human preferences here. Suppose A and B are two outcomes, $\varepsilon$ a positive amount, and $p$ a probability that $0 \leq p \leq 1$, then

a) If $\mathrm{A}$ is preferred to $p \mathrm{~B}, \mathrm{~A}+\varepsilon$ is preferred to $p \mathrm{~B}$; but $\mathrm{A}+\varepsilon$ is not necessarily preferred to $p\left(\mathrm{~B}+\frac{\varepsilon}{P}\right)$;

b) If $p \mathrm{~B}$ is preferred to $\mathrm{A}, \quad p(\mathrm{~B}+\varepsilon)$ is preferred to $\mathrm{A}$; but $p(\mathrm{~B}+\varepsilon)$ is not necessarily preferred to $\mathrm{A}+p \varepsilon$.

This indicates that an increment of benefit on the option favored would firm up the chooser's preference, 
while increments on both sides don't.

\subsection{Misuse of Sample Mean}

Studies that use statistical methods require appropriate understanding of statistics used. When we require subjective certainty equivalents for lotteries out of subjects, we must make sure that the subjects understand what mathematical expectation is because it would be unfair otherwise to use the statistic that subjects don't understand later as a criterion testing subjects' preferences. More importantly, we should sure that we experimenters ourselves fully understand the statistics we are using.

However, there exists the logic leap from existence of statistic to apprehension of statistic. Just the fact that the statistics are invented doesn't mean they could be fully understood. Typical example here is, we are of the opinion, the basic and long been misused statistic of average.

Average, arithmetic or compound, is a basic statistic that is used everywhere as a estimator of general standard. Broadly as it has long been used, human mind could hardly grasp what an average really is. When it is said that Class A bares an average score of 75 in a mid-term examination, it is interpreted immediately by human mind, or at least System 1, that almost every student in Class A got test results in the neighborhood of 75, which is obviously erroneous. And when the second news came that Class B has an average score of 82, again it is translated into a statement that almost every student in Class B got a better score than students in Class A. Ludicrous as it may sound, and despite almost everyone would deny these kinds of thoughts had ever come to them and they really understand what is an average, the very logical fallacy is repeated frequently in studies of human preferences, especially in those which experiments involving subjects are conducted.

The common use of average is like this: conduct the experiment to the subjects, have their responses documented, average the answers and analyze the average answer as a supposed individual who represents the whole sample. The basic idea of using average is to average out unwelcome volatility and thus get a general trend. But this could be perilous in studying behavioral facts of human preferences, for in these kinds of studies, what we need is exactly the opposite: human preferences, such as risk preferences, are very volatile, to say nothing of the fact that an individual's preference vary under arbitrary situations. And this very divergence in preferences is exactly what worth investigating. This is not to say average should be abandoned in preferences analysis. As a matter of fact, one can never avoid using it because it would be invalid and unnecessary to focus on every individual. However, we can revise the sample mean method by dividing the whole sample into groups with obviously different characteristics, thus saving these divergences from being willy-nilly averaged out. We are to employ this method into our experiment in Part III.

Failure of human minds' apprehension of other statistics, such as expected value are entertainingly discussed by Taleb [12], where he asked readers to imagine a combination of pleasure of a vacation that will happen $85 \%$ in Paris and 15\% in Caribbean. In fact, he asserted, that "[our] brain can properly handle one and only one state at once - unless [you] have personality troubles of a deeply pathological nature".

\section{Risk Preference Families}

So far we have shown that the linkage of the two branches of the value function and the use of total average in studying human preferences are mistakable. And we claimed in Section II that not only risk preferences should be studied separately in gain and loss domain, even in the domains of gain or loss per se, they cannot be simply generalized as "risk averse" or "risk seeking". Our experiment following is to test the different risk preferences under gambles of different prize level.

\subsection{The Experiment and Results}

The process employed a two-stage method proved unbiased by Becker et al. [13]. But rather than eliciting subjective certainty equivalents under different probability to win, we require certainty equivalents under different prizes and losses at a same probability $50 \%$. Prizes and losses provided in the experiment ranged from 2 to $1000000(2,6,10,15,25,30,50,100,200,400,750$, $1000,5000,100000,1000000)$. The experimental instructions are written in English. We assume this to be a semi-"back translation" (a concept put forward by Brislin [14]) for we, the designers, are Chinese).

The reason why we didn't divide the probability into smaller minor unity is that we fear that gradual increases in probability make people confused and thus inducing mindless answers. We assume that people get vague idea about the difference between chances of $30 \%$ and $40 \%$, but they surely feel different among a half chance to win, less than a half to win, and more than a half to win. Hence we choose $50 \%$ as our constant probability, presuming that our subjects stay cognitively conscious during the test.

Note that all subjects are students major in either statistics, actuarial science, risk management, or financial engineering, hence it is reasonable to assume than they know quite well the concept of mathematical expectation, justifying our using it later as a criterion in discussing 
risk preferences.

To rule out the majority heuristic and the misuse of total average discussed in Part II, we employ a different method in interpreting the results. Risk preferences toward possible gains and losses are discussed separately. We are to show the risk preferences of gain domain and those of loss domain, but we don't and cannot provide a single type of risk preference that summarizes the whole picture. We avoid believing in average of the total. Rather, responses are categorized, according to their characteristics, into groups. In fact, we intuitively expected, rather than predicting, a general but nothing specific trend before we get the results: respondents may show a tendency toward risk seeking when the possible prizes/losses are small, and they may tend to be risk averse when the possible prizes/losses surge to certain higher levels. However, we didn't plan to derive the general trend which represents the average (and we don't believe any single one could represents all); instead, we focus on detecting different patterns of risk preferences among subjects. We even tried to analyze anomalies among the results and explain why they are like that. Defects in our experiment is that it provided no real money rewards. Since we are trying to elicit subjects' preference under big prize, we cannot afford to actually pay them what they won (For experiment that involving real subjects losses, see Etchart-Vincent and l'Haridon [15]). But they are informed that rewards are to be paid according to their final results. Subjects are urged to behave as if the gambles are real and independent throughout the experiment.

We used in the gain domain the ratio of certainty equivalent to expected value, or $\mathrm{CE} / \mathrm{E}$, as major criterion of subjects risk preferences. If $\mathrm{CE} / \mathrm{E}$ of a single response exceeds unity, the subject is said to show a sign of risk seeking toward the gamble, while if $\mathrm{CE} / \mathrm{E}$ is lower than one, it signals risk averse. In the loss domain, on the other hand, the ratio of maximum premium (which means that the maximum certain quantity the subject is willing to pay to eliminate the risk of suffering potential loss) to expected value, or $\mathrm{MP} / \mathrm{E}$, is used so that if $\mathrm{MP} / \mathrm{E}$ of a response toward a gamble exceeds one, it shows risk averse, and if MP/E is less than one, it shows risk seeking. For example, if the subject is extremely risk seeking toward a potential loss, then she is not willing to pay even a penny to an insurer to have her asset underwritten. In this case, the maximum premium will be 0 , thus MP/E is equal to 0 .

For the purpose of illustrating the misuse of total average that has been discussed in Part II, Figure 3 manifests the total average $\mathrm{CE} / \mathrm{E}$ and $\mathrm{MP} / \mathrm{E}$ values under the prize/loss series toward gains and losses respectively. It seems obvious, from the graphs, that there is a trend of decreasing $\mathrm{CE} / \mathrm{E}$ ratio and increasing $\mathrm{MP} / \mathrm{E}$ ratio. What's

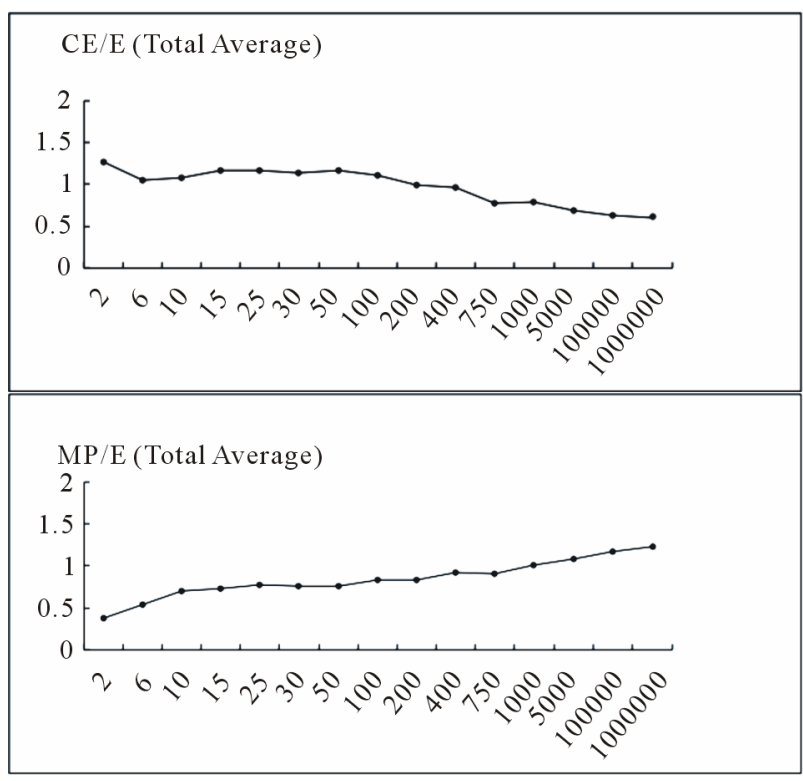

Figure 3. Total average of $\mathrm{CE} / \mathrm{E}$ ratio and MP/E ratio.

more, average $\mathrm{CE} / \mathrm{E}$ ratio of our experiment rarely exceeds unity conspicuously and so does MP/E ratio. This indicates that except for extremely small prizes (such as $50 \%$ to win 2) and extremely big losses (such as $50 \%$ to lose 1000000), respondents show preferences of risk averse in gain domain and risk seeking in loss domain, consistent with what is told by prospect theory and its value function.

We categorized according to their features the final results of gain domain and loss domain respectively into three families. As is shown by Figure 4 some typical individual responses, these categories are named "General", "Arch", and "Bounce" in gains, and "General", "Neutral" and "Seeking" in the losses. Do notice that these results are not paired with each other and must be viewed separately, which means that, for instance, a subject in "General" family of gain domain can either be in "General", "Neutral" or "Seeking" in the loss part.

The first three captures of Figure 4 manifests three preference families that we detected from the subjects in questions involving possible gains. "General" family shows a preference pattern that fits the majority of the respondents, in which agents show risk seeking toward relatively small prizes, and they gradually become risk averse as the prize become more and more attractive. Note that although "General", like the total average CE/E, decreases as the prize level sour, the line does not fall below unity until the prize level reaches somewhere around 200 , whereas the total average $\mathrm{CE} / \mathrm{E}$ rarely surpasses one. Respondents in "Arch" family typically start from strict risk averse, then becomes increasingly risk seeking, but when the prize level seems attractive enough to them, they show a sign of risk averse again. The $\mathrm{CE} / \mathrm{E}$ 


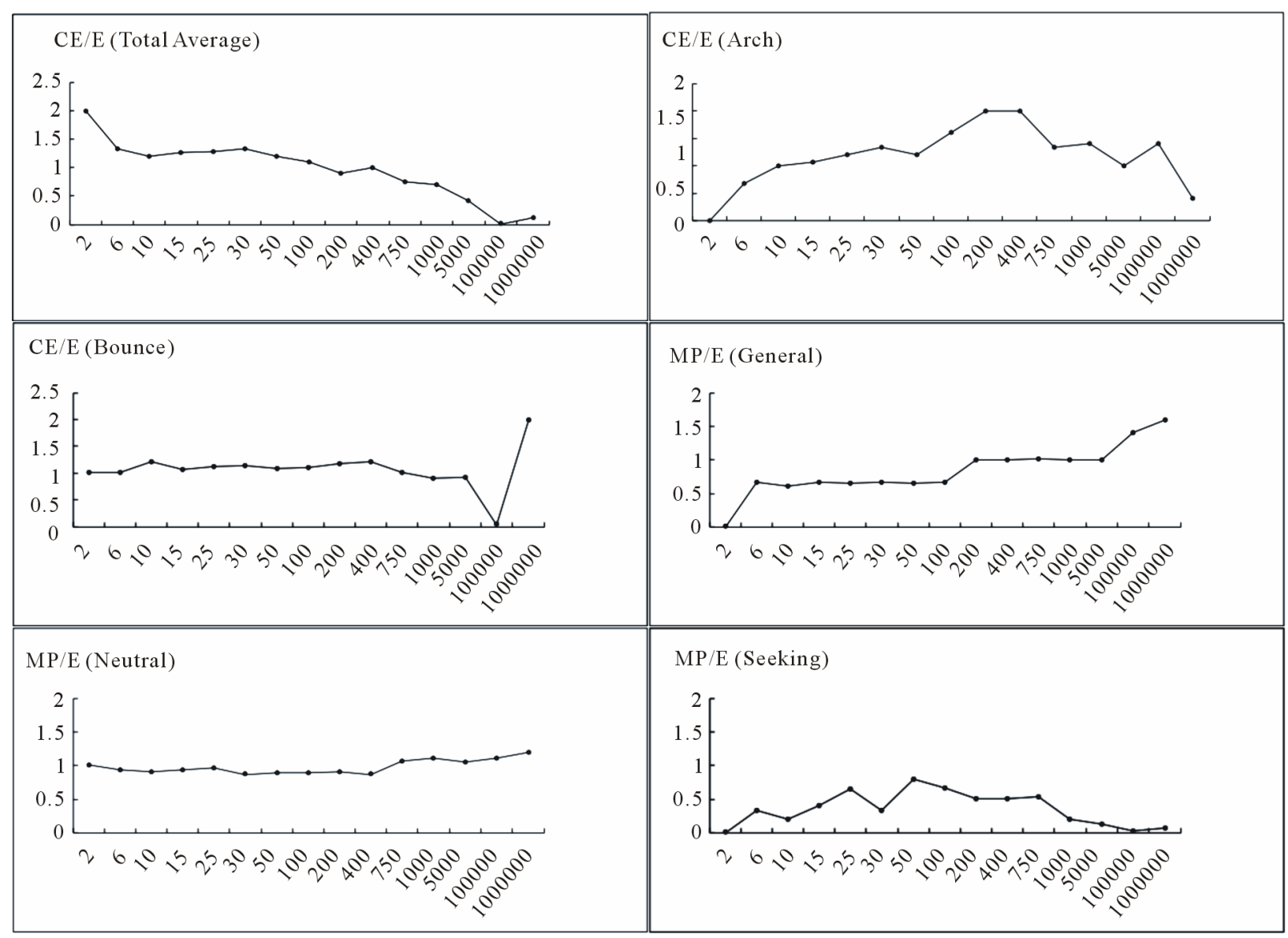

Figure 4. Categorizing of final results in gain and loss domains.(1.General)

line of this family crosses the unity twice. Subjects in "Bounce" family, albeit not large in number, shows an interesting and sharp risk preference reversal when the prize level gets extremely high, before which they generally share risk preferences with those in "General" family.

The last three captures, on the other hand, shows the three risk preference families of the loss domain. Agents in "General (Loss)" family are shown to have increasing $\mathrm{MP} / \mathrm{E}$ ratio as the loss level approaches extreme. But note that MP/E values of a typical respondent in "General (Loss)" family exceeds unity when the possible loss level reaches 200 or so, while in total average ratios, $\mathrm{MP} / \mathrm{E}$ only goes beyond 1 when the possible loss becomes astronomical. "Neutral" family possesses MP/E ratio hovering around one whatever the possible loss would be. Finally, subjects in "Seeking" family have $\mathrm{MP} / \mathrm{E}$ ratio always below unity; they are always risk seeking, tending to run the risk of losing.

Counterintuitive finding of our experiment is that risk preferences of gain and loss domains barely cross paths. For example, not a single one of the subjects showed a sign of risk neutral in gains part, and no one in losses part presented a sudden preference reversal as is in
"Bounce" family. We find that subjects behaved sharply different toward risks of certain gains and losses, and their psychologies, influenced by various factors, varied individually and were very volatile.

We interviewed after the experiment some of the subjects and asked for what were their psychologies when providing answers. To our surprise, every subject interviewed gave exactly the same reason justifying their subjective certainty equivalent toward small prized and losses (such as a $50 \%$ to win/lose 2), which goes like: The possible gains/losses are so small that I don't care if I win or lose them. But their explanations diverse. For those who have CE/E ratio of 2 or bigger toward possible gain, their explanations were typically "I don't care about the little gain so that I just gamble. Either win or not win, no big deal"; for those who have CE/E ratio of smaller value, their justifications were like "I don't care the winning of the little amount of money". In the loss part, none of the subjects has high MP/E ratio toward small possible loss; they chorused a preference of risk seeking, with MP/E ratios ranging from 0 to 1 . Similarly, they asserted in the aftermath interview that they "answered so because they didn't care about the small 
losses". Rationally speaking, if subjects "don't care" whether they gamble or not, namely they think themselves indifferent between gambling or not gambling, that should be interpreted as risk neutral thus their subjective certainty equivalent should be equal to the mathematical expectation, entailing a $\mathrm{CE} / \mathrm{E}$ or $\mathrm{MP} / \mathrm{E}$ ratio of 1 . However, the data just showed that behavior of most subjects wasn't consistent with this rationality. Thus we have reason to doubt that when $\mathrm{CE} / \mathrm{E}$ or $\mathrm{MP} / \mathrm{E}$ ratio does equal to unity, that doesn't necessarily mean the subjects are risk neutral toward the gamble.

The sudden $\mathrm{CE} / \mathrm{E}$ ratio bounce back of subjects in "Bounce" family reflects gambler's psychology. When respondents in the family were asked why they suddenly wanted to take risk in the high prize gamble, they typically answer that when the prize level became extremely high, the lottery turns into a gamble. And a gamble is a gamble, either one win the big, or walk away with nothing.

Most subjects in "Neutral" family of loss domain said they feel horrible on the thought of losing their money, be it the premium they pay or the money they lose if they choose to gamble. Rather than endless dithery, they simply chose to stay indifferent: write down certainty equivalents entered around mathematical expectations, and whatever happens is destiny.

Agents in "Seeking" family of loss domain usually assert that they would rather run the risk of losing the gamble than paying anything to eradicate the risk, so that their fortunes still have a good $50 \%$ chance to stay intact. Although we highly doubt that this kind of psychology is resulted partly from the fact that the possible losses in the experiment are hypothetical.

\subsection{Models}

Kachelmeier and Shehata (1992) introduced a linear regression model that includes subject effects, incorporating correlated errors entailed by individual differences in risk preferences. Our models bases essentially on the Kachelmeier and Shehata's CERATIO model but a) we ignore the percent effects for lotteries in our experiment share the constant winning/losing chance of $50 \%$, b) we used logarithm of prizes/losses in the model and c) we introduce a similar MPRATIO model for MP/E ratios with logarithm of prize factor replaced by logarithm of loss. Our regression models are shown as followed:

$$
\begin{aligned}
\text { CERATIO }= & \alpha+\beta_{1} \log (\text { Prize }) \\
& +\sum_{j=1}^{n-1} \beta_{2 j} \text { Subject }_{j}+\varepsilon \\
\text { CERATIO } & =\alpha^{*}+\beta_{i}^{*} \log (\text { Loss }) \\
& +\sum_{j=1}^{n-1} \beta_{2 j}^{*} \text { Subject }_{j}+\varepsilon
\end{aligned}
$$

where CERATIO and MPRATIO respectively means ratio of certainty equivalent to expected value and ratio of maximum premium to expected value; $\alpha$ and $\alpha^{*}$ are intercepts of regressions; $\log$ (Prize) and $\log$ (Loss) are logarithm of Prizes and Losses series in the lotteries;

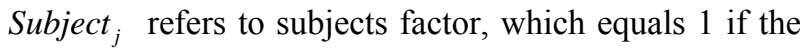
observation is from the $j$ th observation, -1 if from the $n$th observation, and 0 otherwise; $\varepsilon$ is the disturbance.

The regression coefficients and the results of tests are tabulated as follows.

Where CONSTANT $=$ the intercept parameter;

$S_{i}=$ the $j$ th subject

$p^{*}=$ logarithm of Prize

Advantage of categorizing risk preference into families, and thus deriving the categorized regression models, could be observed first in terms of the increase in both $\mathrm{R}$ square, namely the goodness of fit, and adjusted R square in both "General" families of gain and loss parts as is shown by Table 3 and Table 7, compared to that of the sample average data in Table 2 and Table 6. R square increases from 0.433 in sample average to 0.758 in "General" family of gain domain, and from 0.784 in sample average to 0.839 in "General" family of loss domain. And the corresponding adjusted R square as well increase from 0.392 to 0.533 and from 0.586 to 0.683 respectively. Besides, estimates derived by OLS technique in CERATIO and MPRATIO model show statistical significance by and large, as can be seen particularly in Table 3, Table 5, Table 7, and Table 8. Table 4 and Table 9, corresponding to "Arch" family of Gain Domain and "Seeking" Family of Loss Domain, show insignificance in estimates of parameters; we attribute this insignificance to the small sample size, and it can be that they are not representative to the population.

Second, in most families, the variances of estimates of both CERATIO and MPRATIO drop from original variances of estimates of CERATIO and MPRATIO in sample average regression models, indicating better stability. See Table 10.

\section{5. "D" for Testing Validity of Sample Average}

It is mentioned without evidence in Sec II.C that the use of total average in analyzing behavioral facts of human preferences is mistakable for it averages out not only unwelcome volatility of the data but also the divergence between subjects preferences which worth investigating. This is observable from Figure 3 and Figure 4, especially in the gain part, for the three risk preference families, "General", "Arch", and "Bounce", viz., of gain domain bear sharply different shaped $\mathrm{CE} / \mathrm{E}$ lines.

To test whether the use of sample average averages 
Table 2. Results of CERATIO model for sample average of gain domain.

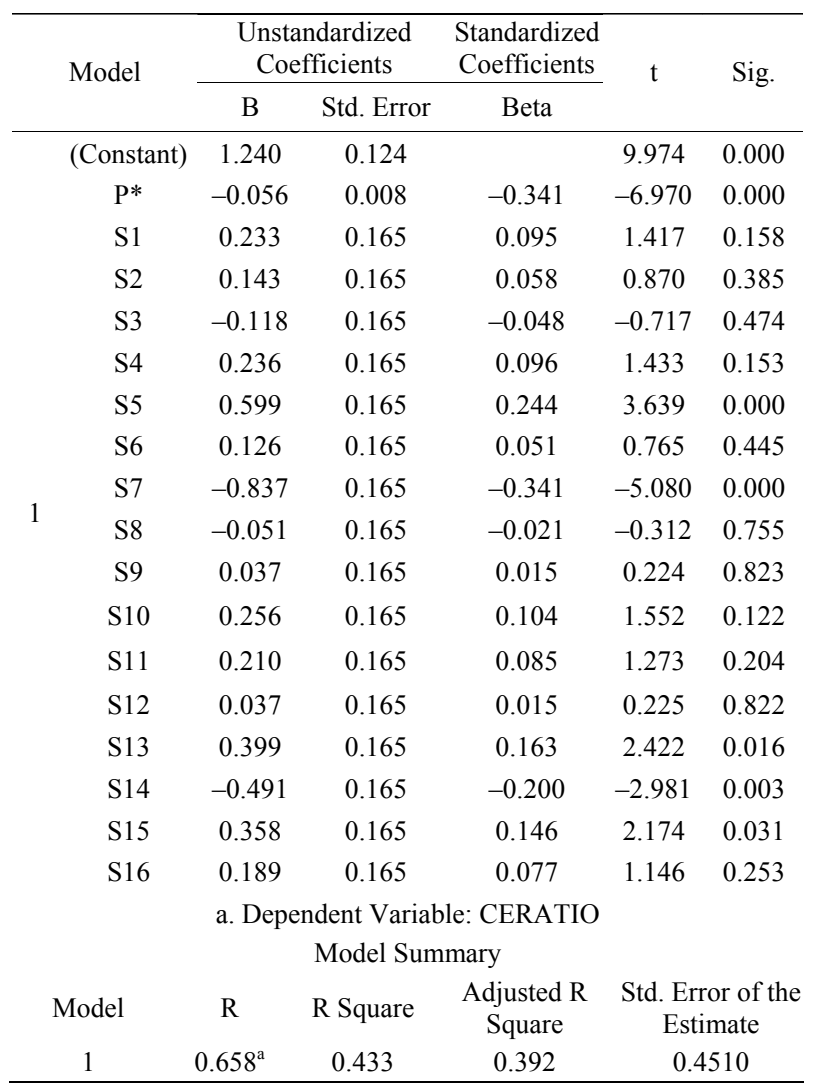

a.Predictors: (Constant), S16, P*, S5, S2, S1, S4, S3, S8, S7, S6, S9, S10, $\mathrm{S} 12, \mathrm{~S} 11, \mathrm{~S} 13, \mathrm{~S} 14, \mathrm{~S} 15$.

Table 3. Results of CERATIO model for "general" family of gain domain.

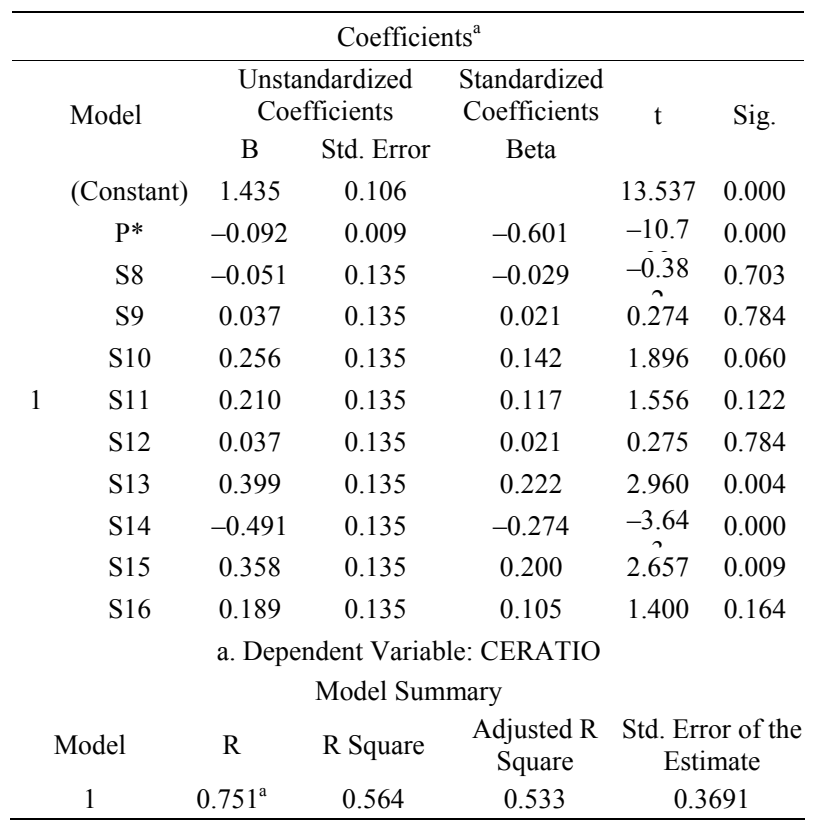

a.Predictors: (Constant), S16, P*, S15, S14, S13, S12, S11, S10, S9, S8.
Table 4. Results of CERATIO model for "arch" family of gain domain.

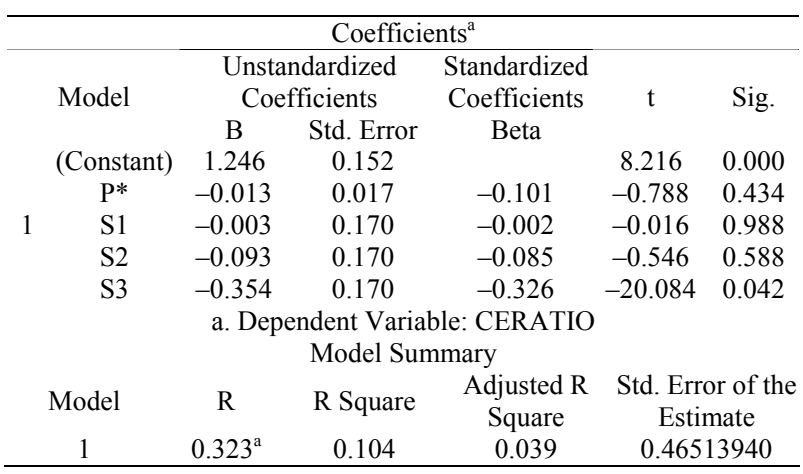

a.Predictors: (Constant), S3, P*, S2, S1.

Table 5. Results of CERATIO model for "bounce" family of gain domain.

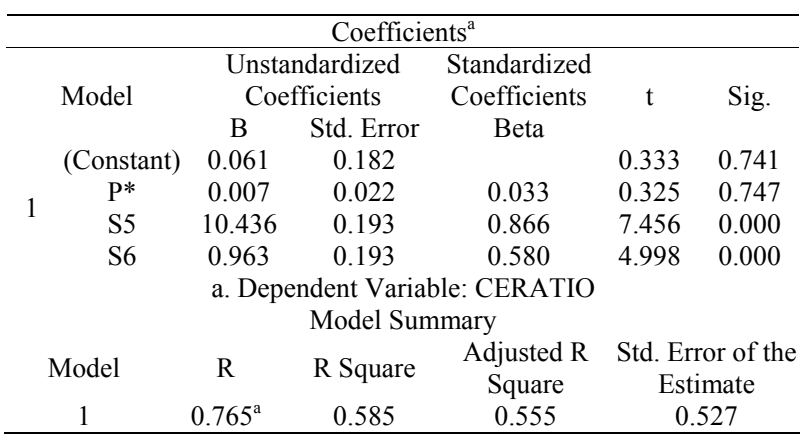

a.Predictors: (Constant), S6, P*, S5.

Table 6. Results of MPRATIO model for sample average of loss domain.

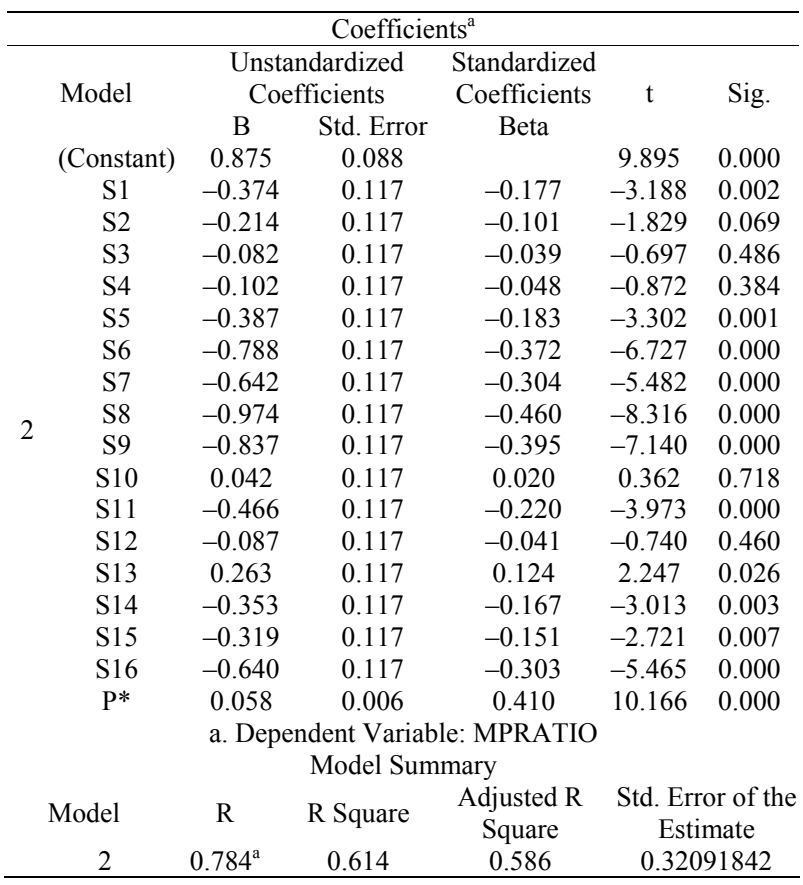

a.Predictors: (Constant), P*, S16, S5, S2, S1, S4, S3, S8, S7, S6, S9, S10, S12, S11, S13, S14, S15. 
Table 7. Results of MPRATIO model for "general" family of loss domain.

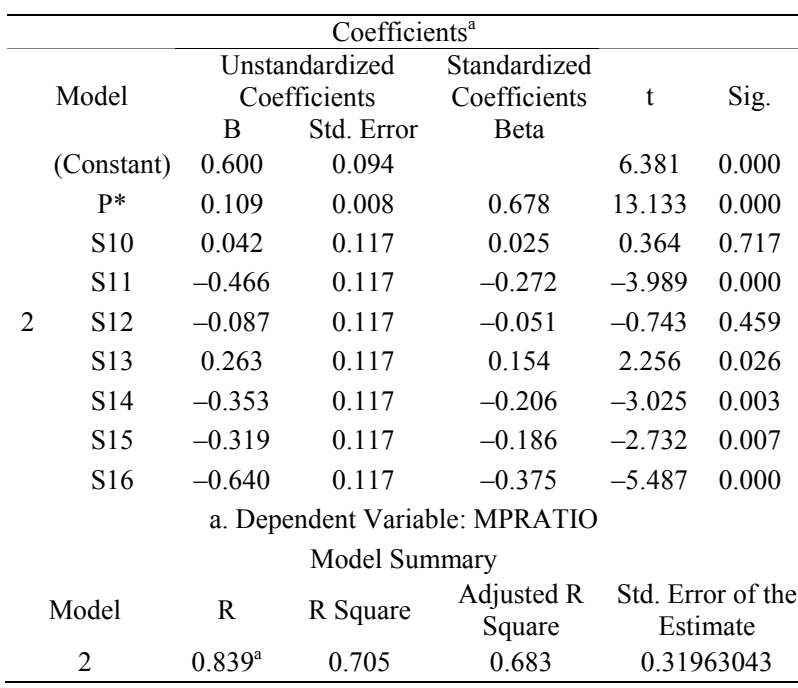

a.Predictors: (Constant), S16, P*, S15, S14, S13, S12, S11, S10.

Table 8. Results of MPRATIO model for "neutral" family of loss domain.

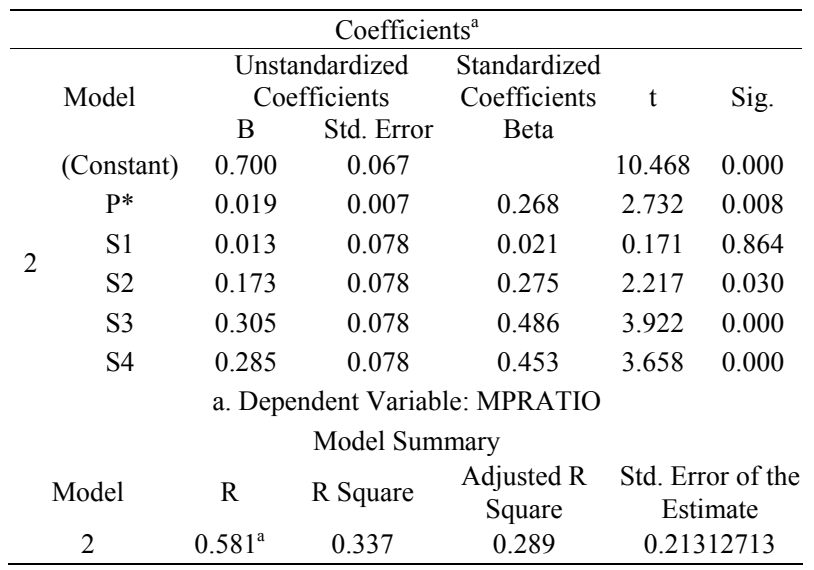

a.Predictors: (Constant), S4, P*, S3, S2, S1.

Table 9. Results of MPRATIO model for "seeking" family of loss domain.

\begin{tabular}{|c|c|c|c|c|c|c|}
\hline \multicolumn{7}{|c|}{ Coefficients $^{\mathrm{a}}$} \\
\hline \multirow{2}{*}{\multicolumn{2}{|c|}{ Model }} & \multicolumn{2}{|c|}{$\begin{array}{l}\text { Unstandardized } \\
\text { Coefficients }\end{array}$} & \multirow{2}{*}{$\begin{array}{c}\text { Standardized } \\
\text { Coefficients } \\
\text { Beta }\end{array}$} & \multirow[t]{2}{*}{$\mathrm{t}$} & \multirow[t]{2}{*}{ Sig. } \\
\hline & & B & Std. Error & & & \\
\hline & (Constant) & 0.326 & 0.071 & & 4.601 & 0.000 \\
\hline \multirow{4}{*}{2} & $\mathrm{P}^{*}$ & 0.005 & 0.008 & 0.074 & 0.635 & 0.528 \\
\hline & S6 & 0.048 & 0.079 & 0.087 & 0.609 & 0.545 \\
\hline & S7 & 0.194 & 0.079 & 0.350 & 2.448 & 0.018 \\
\hline & S8 & -0.138 & 0.079 & -0.248 & -1.736 & 0.088 \\
\hline \multicolumn{7}{|c|}{ a. Dependent Variable: MPRATIO } \\
\hline \multicolumn{7}{|c|}{ Model Summary } \\
\hline & Iodel & $\mathrm{R}$ & R Square & $\begin{array}{l}\text { Adjusted R } \\
\text { Square }\end{array}$ & \multicolumn{2}{|c|}{$\begin{array}{l}\text { Std. Error of the } \\
\text { Estimate }\end{array}$} \\
\hline & 2 & $0.499^{\mathrm{a}}$ & 0.249 & 0.195 & \multicolumn{2}{|c|}{0.21731274} \\
\hline
\end{tabular}

a.Predictors: (Constant), S8, P*, S7, S6.
Table 10. SDs of CERATIO and mpratio in different models.

\begin{tabular}{ccccc}
\hline & Gain average & Arch & Bounce & General \\
\hline$\hat{\sigma}$ & 0.4510 & 0.4651 & 0.527 & 0.3691 \\
& Loss average & Neutral & Seeking & $\begin{array}{c}\text { General } \\
\text { (Loss) }\end{array}$ \\
$\hat{\sigma}$ & 0.3209 & 0.2131 & 0.2173 & 0.3196 \\
\hline
\end{tabular}

out volatility or divergence, namely to test whether the use of sample average is desirable, one should look into the difference between estimates of target values, in this paper the CERATIO and MPRATIO, before and after the sample is categorized into families.

We here focus on the difference between the "distance" between estimates of CERATIO/MPRATIO using categorized models (the families models) and sample average model to the real sample average.

Define Distance Function, denoted DF, which refers to the distance between regression model estimates of CERATIO/MPRATIO to the real sample average of CERATIO/MPRATIO:

$$
\begin{aligned}
& D F=\sum_{i=1}^{15} \sum_{j=1}^{n}\left(\text { CERATIO }_{i, j}{\overline{\text { ERATIO }_{i}}}^{2}\right. \\
& D F=\sum_{i=1}^{15} \sum_{j=1}^{n}\left(\text { MPRATIO }_{i, j} \overline{\text { MPRATIO }}_{i}\right)^{2}
\end{aligned}
$$

where $n=$ subjects sample size;

$\mathrm{CERATIO}_{i, j}=$ regression model estimate of $\mathrm{CE} / \mathrm{E}$ ratio of the $j$ th subject, towards the $i$ th lottery;

$\overline{\mathrm{CERATIO}}_{i}=$ the sample average of CE/E ratio towards the ith lottery and similarly are the meanings of MPRATIO $_{i, j}$ and $\overline{\operatorname{MPRATIO}}_{i}$.

Define $D$ as:

$$
D F=\left(\sum_{i=1}^{S} D F_{i} / n\right)^{1 / 2}
$$

where $s=$ number of families (in this paper three, for gain and loss parts respectively);

$n=$ subjects sample size;

$D F=$ the value by Distance Function in (3) and (3)', then the difference between Ds of uncategorized model and categorized models, denoted $\Delta D$, is the "Averageout Degree" of the sample average regression model, measuring the difference between estimates of the CERATIO and MPRATIO before and after the sample is categorized into families. Thus the averageout is more desirable,which means that it is more likely the use of sample average averages out unwelcome volatility rather than individual divergences, if the absolute value of $\Delta D$, the averageout degree, is more close to zero.

For brevity, here the process of calculations are skipped. Results are shown in Table 3 below:

Note that in the gain realm, the averageout degree, 
the $\Delta D$, as is shown by Table 11, of the test bears a value of great bigger magnitude than that of the loss realm, consistent with our arguments earlier in this section that the use of sample average in the gain domain here might have averaged out not only unwelcome volatility, but also individual divergences. However, it is still premature for us to assert that the use of sample average, the uncategorized model, is undesirable, because to do that one need a benchmark $\Delta D$ level upon with one could judge the desirability of the use of uncategorized sample average, which needs further studies.

\section{Conclusions}

There is no such a single function that can represent the volatile and divergent preferences of mankind. The prospect theory value function mistakably links its two branches in gain and loss domains together, which, albeit right intuitively, falls in the pit in the majority heuristic. Also, using sample mean in analyzing data in the studies of behavioral economics could be perilous because this method treats the whole sample as an individual and willy-nilly presumes that the average preference could stand for the whole, while in studying the behavioral facts of human preferences, individual divergences are pivotal and worth deep investigation. We proposed in the paper an approach toward modeling and data analyses, which involves categorizing the sample into different preference families and developing the sub-regression models. We suggested that this approach is statistically more reliable than the mere analysis of the sample means. But we should also point out that our theory still is based on experiments. It provides a way of revealing the fragility of uncategorized regression model in behavioral studies, but it is incapable of testing the validity of using

Table 11. DF, $D$, and $\Delta D$ values of the test.

\begin{tabular}{ccc}
\hline & DF uncategorized & DF categorized \\
\hline Gain-Arch & 2.030457834 & 3.214633228 \\
Gain-Bounce & 17.76499969 & 19.21976959 \\
Gain-General & 10.02344834 & 12.43379891 \\
D & 1.324406768 & 1.432155959 \\
Loss-Neutral & $\Delta D$ & 0.107749191 \\
Loss-Seeking & 82.30766511 & 4.105989609 \\
Loss-General & 61.35279015 & 18.68931585 \\
D & 3.229913867 & 154.5656861 \\
& $\Delta D$ & 3.230015403 \\
\hline
\end{tabular}

experiments in behavioral economics, which, we believe, calls for further philosophical discussion.

\section{Reference}

[1] Kahneman, Daniel. and Tversky, Amos., "Prospect Theory: An Analysis of Decision under Risk", Econometrica, Vol. 47, No. 2, 1979, pp. 263-92

[2] Odean, Terrance., "Are Investors Reluctant to Realize Their Losses", The Journal of Finance, Vol. 53, No. 5, 1998, pp. 1775-98

[3] Thaler, Richard H., and Johnson, Eric J., "Gambling with the House Money and Trying to Break Even: The Effects of Prior Outcomes on Risky Choice", Management Science, Vol. 36, No. 6, 1990, pp. 643-60

[4] Thaler, Richard H., "Mental Accounting and Consumer Choice", Marketing Science, Vol. 4, No.3, 1985, pp. 199-214

[5] Kahneman, Daniel., and Riepe, Marke W. "Aspects of Investor Psychology: Beliefs, preferences, and biases investment advisors should know about", Journal of Portfolio Management, Vol. 24, No. 4, 1998, pp. 67-91

[6] Kachelmeier, Steven J. and Shehata, Mohamed., "Examining Risk Preferences Under High Monetary Incentives: Experimental Evidence from the People's Republic of China", The American Economic Review, Vol. 82, No. 5, 1992, pp. 1120-41

[7] Tversky, Amos. and Kahneman, Daniel., "Advances in Prospect Theory: Cumulative Representation of Uncertainty", Journal of Risk and Uncertainty, No. 5, 1992, pp. 297-323

[8] Staw, B. M. "The Escalation of Commitment to a Course of Action," Acad. Management Rev., Vol. 6, No. 4, 1981, pp. 577-87

[9] Laughhunn, D. J. and J. W. Payne. 1984. "The Impact of Sunk Outcomes on Risky Choice Behavior," INFOR (Canad. J. Per. Res. and information Processing), No. 22, 1984, pp. 151-81

[10] Tversky, Amos. and Kahneman, Daniel., "Judgement under Uncertainty: Heuristics and Biases", Science, New Series, Vol. 185, 1974, pp. 1124-31

[11] Fredrick, Shane., "Cognitive Reflection and Decision Making”, The Journal of Economic Perspectives, Vol. 19, No. 4, 2005, pp. 25-42

[12] Taleb, Nassim N. "Fooled by Randomness: The Hidden Role of Chance in Life and in the Markets", W. W. Norton, ISBN-10: 1587990717, October 2001, pp. 182-83

[13] Becker, G. M., DeGroot, Morris H., and Marschak, Jacob, "Measuring Utility by a Single-Response Sequential Method," Behavioral Science, No. 9, 1964, pp. 226-32.

[14] Brislin, Richard W., "Back-Translation for Cross-Cultural Research," Journal of Cross-Cultural Psychology, No. 1, 1970, pp. 185-216

[15] Etchart-Vincent, N. and l'Haridon, O., "Monetary Incentives In the Loss Domain and Behavior Toward Risk: An Experimental Comparison of Three Reward Schemes In- 


\section{Appendix: Replication Experiment of Pros- pect Theory Problems}

PROB1: In addition to whatever you own, you have been given 1,000 . You are now asked to choose between
A: $50 \%$ chance to win 1,000
B: a sure gain of 500

PROB2: In addition to whatever you own, you have been given 2,000. You are now asked to choose between
A: $50 \%$ chance to lose 1,000

B: a sure loss of 500 PROB3: Choose between:

A: $25 \%$ to win 6,000

B: $25 \%$ to win 2,000 and another $25 \%$ to win 4,000 PROB4: Choose between:

A: $25 \%$ to lose 6,000

B: $25 \%$ to win 2,000 and another $25 \%$ to lose 4,000 